\title{
Domain-Integrated Field Equations Approach to Static Magnetic Field Computation-Application to Some Two-Dimensional Configurations
}

\author{
Adrianus T. de Hoop and Ioan E. Lager
}

\begin{abstract}
The recently developed domain-integrated field equations approach to static magnetic field computation is applied to some two-dimensional configurations, viz. a model rotor/stator slot configuration and a model magnetic recording head configuration. Consistently linear edge expansion functions are used for the magnetic field strength, together with consistently linear face expansion functions for the magnetic flux density, both on a triangular mesh. The method handles large contrasts in material properties on a coarse mesh, with excellent global error properties, in acceptable computation times.
\end{abstract}

Index Terms-Inhomogeneous media, numerical analysis.

\section{INTRODUCTION}

I N OUR PREVIOUS contribution [1] the domain-integrated field equations approach to static magnetic field computation has been developed and tested on some two-dimensional configurations with a known analytic solution. The method was implemented in a computer code:

- by using consistently linear edge expansions for the magnetic field strength, together with consistently linear face expansions for the magnetic flux density,

- in conjunction with the minimization of the $L^{2}$-norm of the discrepancy in the constitutive relation (both in its passive reaction to the field, via its tensor or scalar permeability, and in its active part, via a prescribed, field independent, source distribution of permanent magnetization).

For simplicity, the test cases in [1] were implemented on a square mesh with bilinear expansions on each element. These examples have proved that the method can handle large contrasts in material properties, on a coarse mesh, with excellent global error properties, in acceptable computation times.

The present contribution begins by outlining the natural manner in which the ideas that are at the core of the domain-integrated field equations approach to static magnetic field computation stem from the underlying physics in strongly heterogeneous (and anisotropic) media. The method is then

Manuscript received October 25, 1999.

A. T. de Hoop is with the Laboratory for Electromagnetic Research, Faculty of Information Technology and Systems, Delft University of Technology, Mekelweg 4, 2628 CD Delft, The Netherlands (e-mail: A.T.deHoop@ITS.TUDelft.NL).

I. E. Lager is with the International Research Centre for Telecommunications-transmission and Radar, Faculty of Information Technology and Systems, Delft University of Technology, Mekelweg 4, 2628 CD Delft, The Netherlands (e-mail: I.Lager@ITS.TUDelft.NL).

Publisher Item Identifier S 0018-9464(00)05487-X. compared with other computational techniques in the realm of static magnetic fields.

For further investigating the performance of the domain-integrated field equations method, a new, improved version of the software package presented in [1] has been developed. The present version implements the method on a triangular mesh, which is the proper mesh to be used. The performance of the method is illustrated by discussing some two-dimensional engineering model configurations, viz. a model asynchronous, electrical, rotary machine rotor/stator slot configuration and a model magnetic recording head configuration. Again, the method proves to provide, in configurations with large contrasts in material properties (our tests go up to a factor of one thousand), on a coarse mesh, results with excellent global error properties, in acceptable computation times. Note that, with the chosen sets of expansion functions, mesh refinement near interfaces across which large contrasts in material properties occur, is superfluous.

\section{The Domain-INTEGRATED FiELD EQUATIONS APPROACH to STATIC MAGNETIC Field COMPUTATION}

\section{A. Physical Justification of the Method}

The domain-integrated field equations method can be viewed upon as to be the computational method that models, as closely as possible, the underlying physics in strongly heterogeneous (and anisotropic) media. Strongly heterogeneous media are characterized by the property that on any scale on which the macroscopic laws of nature maintain to hold, the granular structure persists. Now, in any practical situation one is only interested in the values of the field quantities in such a medium down to the scale where actual measurements are still feasible. Let us denote this scale as the mesoscopic scale (to distinguish it from the microscopic or atomic scale where, for the field description, the quantum laws of physics are required). In view of the fact that on the mesoscopic scale heterogeneity still persists, with its associated discontinuous behavior of (at least some components of) the field quantities involved, the field quantities, as such, do not belong to the class of spatially differentiable functions and, hence, corresponding field equations in differential form cannot serve as a point of departure. On the mesoscopic scale, the domain-integrated field equations are the alternatives to serve as the basis for field computation. In the latter, only the field quantities themselves occur, whose values should, on physical grounds, remain bounded and, 


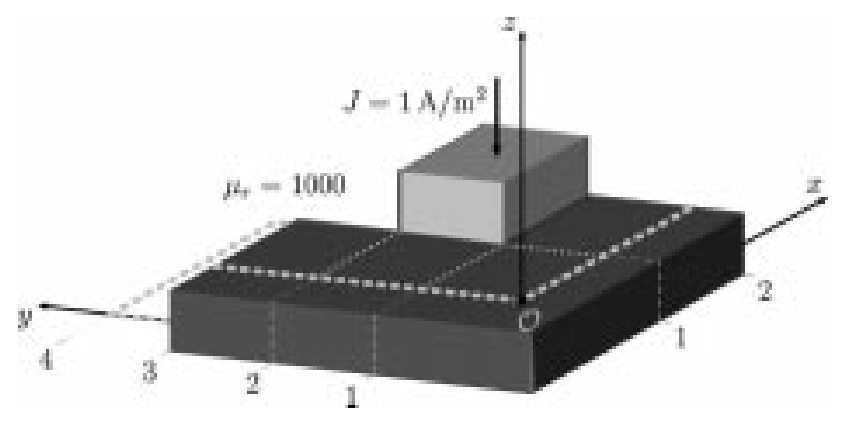

Fig. 1. The Model Rotor/Stator Slot Configuration (all dimensions are in meter). Impressed boundary conditions: $\boldsymbol{n} \times \boldsymbol{H}=\mathbf{0}$ for $\{x=2\}$ and $\{y=4\}$ and $\boldsymbol{n} \cdot \boldsymbol{B}=0$ for $\{x=0\}$ and $\{y=0\}$;

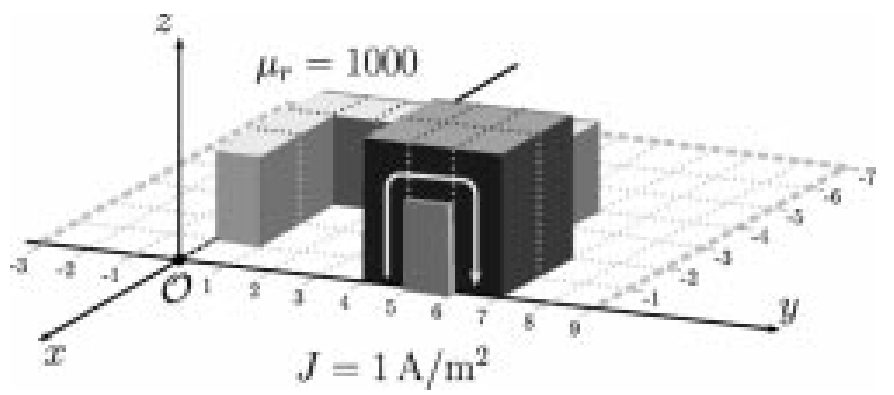

Fig. 2. The model magnetic head configuration (all dimensions are in millimeter). Impressed boundary conditions: $\boldsymbol{n} \times \boldsymbol{H}=\mathbf{0}$ for $\{x=0\}$ and $\boldsymbol{n} \cdot \boldsymbol{B}=0$ for $\{x=-7\},\{y=-3\}$ and $\{y=9\}$;

hence, integrable over any bounding surface of a computational cell of the discretized geometry. A remarkable feature of the domain-integrated field equations is that in them only the field components occur that are continuous across an interface of discontinuity in material properties. For the static magnetic field these are the tangential components of the magnetic field strength $\boldsymbol{H}$ and the normal component of the magnetic flux density $\boldsymbol{B}$. Computationally, we now choose the mesoscopic level such that spatial polynomial expansions of degree one suffice to approximate these continuous components on the boundary surface of any elementary cell. Algebraic topology learns that consistently linear edge expansions (for field quantities whose tangential components are to be continuous) and consistently linear face expansions (for field quantities whose normal components are to be continuous) on a simplicial mesh form a coherent framework in this respect [2].

So far, the physics of the field behavior on a mesoscopic scale dictates the computational formulation of the problem as far as the field equations are concerned. The pertaining constitutive relations present, however, a difficulty of a different nature. Since, by assumption, the medium is on the mesoscopic level still heterogeneous, we cannot assign to the medium a set of (scalar or tensorial) constitutive coefficients that are to relate the magnetic flux density to the magnetic field strength. To model the magnetic constitutive behavior of the medium under consideration, we consistently linearly extrapolate the field quantities from their (edge or face) representations on the surface to the interior of each (simplicial) cell and assume that the extrapolated values allow for the introduction of "smoothed" constitutive coefficients that follow from a minimization procedure applied to the norm in the discrepancy in a constitutive relation that would
TABLE I

COMPUTED VALUES OF INTEGRAL QUANTITIES FOR THE MODEL ROTOR/STATOR SLOT CONFIGURATION

\begin{tabular}{||c|c|c|}
\hline \hline Circulation & $\begin{array}{c}\text { Computed } \\
\text { value }(\mathrm{A})\end{array}$ & $\begin{array}{c}\text { Relative } \\
\text { error (\%) }\end{array}$ \\
\hline $\int_{\Gamma_{1}} \boldsymbol{\tau} \cdot \boldsymbol{H} \mathrm{d} s$ & -0.9911 & 0.89 \\
$\int_{\Gamma_{2}} \boldsymbol{\tau} \cdot \boldsymbol{H} \mathrm{d} s$ & -0.96979 & 3.02 \\
$\int_{\Gamma_{3}} \boldsymbol{\tau} \cdot \boldsymbol{H} \mathrm{d} s$ & -0.96422 & 3.58 \\
\hline \hline
\end{tabular}

\begin{tabular}{||c|c||}
\hline \hline Relative flux per unit length & Computed value \\
\hline $\int_{\Gamma_{4}} \boldsymbol{n} \cdot \boldsymbol{B} \mathrm{d} s / \int_{\Gamma_{\bar{\tau}}} \boldsymbol{n} \cdot \boldsymbol{B} \mathrm{d} s$ & $-1.756 \cdot 10^{-3}$ \\
$\int_{\Gamma_{5}} \boldsymbol{n} \cdot \boldsymbol{B} \mathrm{d} s / \int_{\Gamma_{\bar{\tau}}} \boldsymbol{n} \cdot \boldsymbol{B} \mathrm{d} s$ & $-2.189 \cdot 10^{-3}$ \\
$\int_{\Gamma_{6}} \boldsymbol{n} \cdot \boldsymbol{B} \mathrm{d} s / \int_{\Gamma_{\bar{\gamma}}} \boldsymbol{n} \cdot \boldsymbol{B} \mathrm{d} s$ & $-2.687 \cdot 10^{-3}$ \\
\hline \hline
\end{tabular}

TABLE II

COMPUTATION TIMES vs. MEsh DENSITY FOR THE MODEL Rotor/STATOR SLOT CONFIGURATION

\begin{tabular}{||c||c|c|c|c||}
\hline \hline $\begin{array}{c}\text { Mesh } \\
\text { density }\end{array}$ & $\begin{array}{c}\text { \# degrees } \\
\text { of freedom }\end{array}$ & $\begin{array}{c}\text { \# IC-CG } \\
\text { iterations }\end{array}$ & $\begin{array}{c}\text { Residual } \\
\text { error }\end{array}$ & $\begin{array}{c}\text { Elapsed } \\
\text { time }\end{array}$ \\
\hline $2 \times 4$ & 216 & 2 & $1.0 \cdot 10^{-9}$ & $2 \mathrm{~s}$ \\
$4 \times 8$ & 816 & 4 & $2.2 \cdot 10^{-8}$ & $32 \mathrm{~s}$ \\
$8 \times 16$ & 3168 & 6 & $2.6 \cdot 10^{-8}$ & $1 \mathrm{~m} 32 \mathrm{~s}$ \\
$16 \times 32$ & 12480 & 8 & $1.7 \cdot 10^{-8}$ & $22 \mathrm{~m} 56 \mathrm{~s}$ \\
\hline \hline
\end{tabular}

apply to a locally homogeneous medium. In our case, we have chosen the $L^{2}$-norm for this, but this choice is, of course, open to debate. Since similar arguments hold for prescribed, field independent, source magnetizations $\boldsymbol{M}$, we have followed the same procedure for this quantity.

The procedure indicated above leads to an overdetermined system of linear, algebraic equations in the field expansion coefficients. Such a system has, as we know, no solution. This, in itself, we consider as indicative that we are, again, truly modeling the physics. To substantiate this argument, let us, for a moment, consider the field in a homogeneous piece of matter. Here, we know that the field components are analytic functions of the spatial coordinates, which functions require an infinity of polynomial terms to expand them. Hence, a local representation in the form a polynomial of degree one can, even if sufficiently accurate, only be approximate and never exact. Therefore, the local expansions used cannot (exactly) solve the problem, which property can be rephrased as that the discretized field problem has no solution. The best we can do under such circumstances is to construct the "best" values of the field expansion coefficients in accordance with the physical properties accounted for, i.e. minimizing (subject to a certain error criterion) the discrepancy between the values that are prescribed and the combinations of field expansion coefficients they should be equal to. Here, too, we have chosen for an $\boldsymbol{L}^{2}$-norm . This choice, as well, is subject to debate.

Finally, it is to be remarked that if on the mesoscopic scale the medium should be (piece-wise) continuous, the field quantities in any subdomain of continuity are differentiable vector functions of position and the formulation in terms of field equations in differential form, with a point-wise constitutive relation, holds. Our formulation meets the criterion that, under 


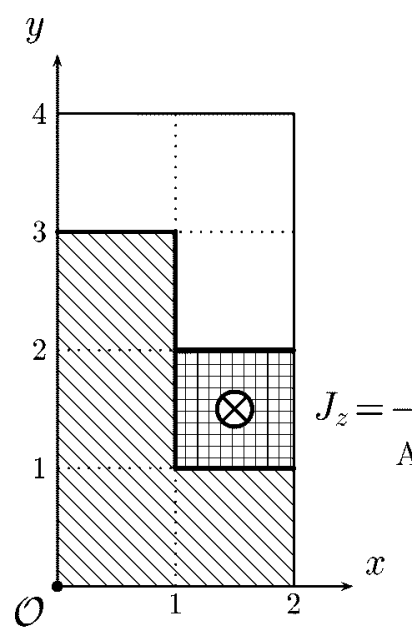

a

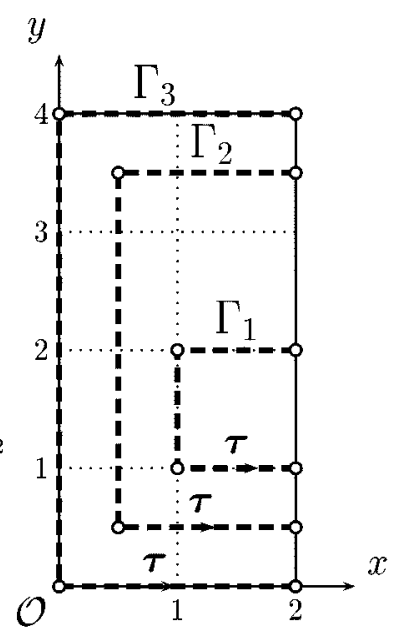

$\mathrm{b}$

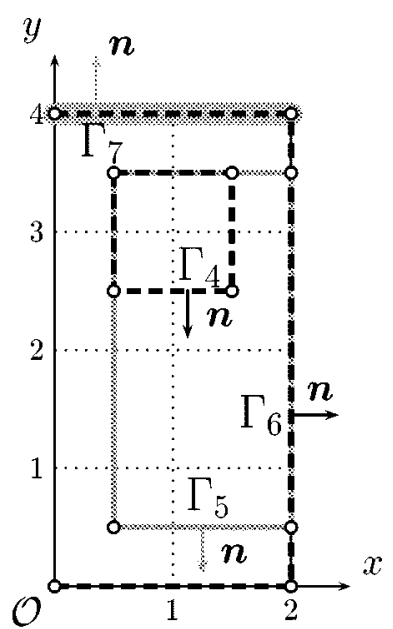

$\mathrm{C}$

Fig. 3. Integration paths for the model rotor/stator slot configuration: (a) The configuration; (b) the integration paths for $\int_{\Gamma_{i}} \tau \cdot H d s$ type integrals; and (c) the integration paths for $\int_{\Gamma_{i}} \boldsymbol{n} \cdot \boldsymbol{B} d s$ type integrals.

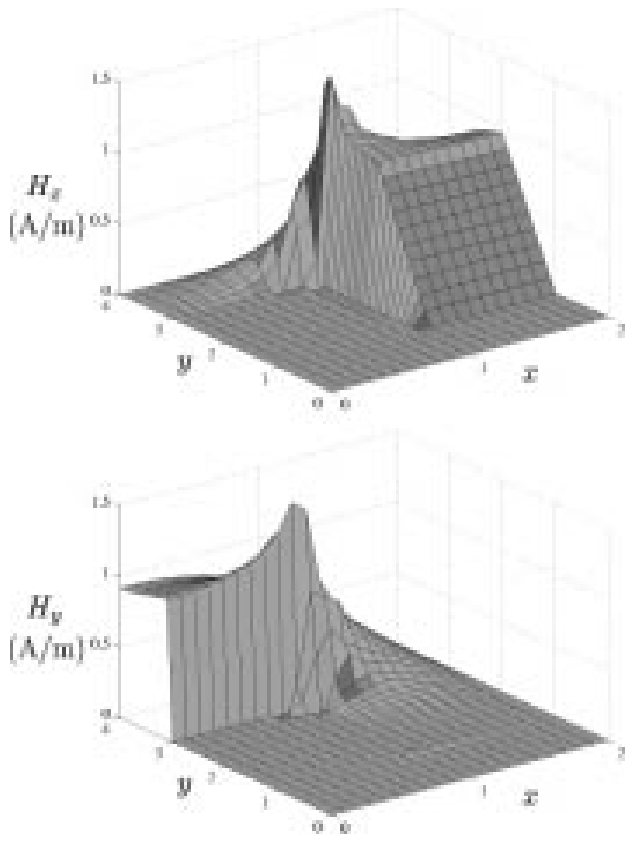

Fig. 4. The plot of the field distribution in the model rotor/stator slot configuration (computational mesh density $8 \times 16$ ).

TABLE III

Computation Times vs. Mesh Density for the Model Magnetic HEAD CONFIGURATION

\begin{tabular}{||c||c|c|c|c||}
\hline \hline $\begin{array}{c}\text { Mesh } \\
\text { density }\end{array}$ & $\begin{array}{c}\text { \# degrees } \\
\text { of freedom }\end{array}$ & $\begin{array}{c}\text { \# IC-CG } \\
\text { iterations }\end{array}$ & $\begin{array}{c}\text { Residual } \\
\text { error }\end{array}$ & $\begin{array}{c}\text { Elapsed } \\
\text { time }\end{array}$ \\
\hline $\begin{array}{r}7 \times 12 \\
14 \times 24\end{array}$ & 2092 & 4 & $4.0 \cdot 10^{-8}$ & $43 \mathrm{~s}$ \\
$14 \times 216$ & 5 & $2.6 \cdot 10^{-8}$ & $10 \mathrm{~m} 2 \mathrm{~s}$ \\
\hline \hline
\end{tabular}

this condition, the equivalence of the domain-integrated field equations formulation and the formulation in terms of field differential equations and a local constitutive relation is evident. Given the relevant conditions, straightforward applications of Gauss' theorem and a continuity argument applied to the constitutive law link the two formulations.

\section{B. Comparison with Other Computational Methods for Static Magnetic Fields}

Nowhere in our analysis the differentiability of the field quantities plays a rôle. This sets the domain-integrated field equations method apart from other methods, in particular from those based on a reformulation of the field problem as a variational one, by a procedure which inherently leans on the use of spatial derivatives of the field vectors.

The domain-integrated field equations method is, arguably, similar to "mixed" formulations, as long as in the latter edge elements are used for expanding $\boldsymbol{H}$ and face elements for expanding $\boldsymbol{B}$. In [3]-[5], however, the additional step consists of a variational formulation, to which our remark above applies. The differentiability condition can be circumvented by the use of differential operators in the weak sense [6, p. 41] for formulating the field equations, but accounting for the constitutive relation still requires the assumption of the differentiability of the field quantities at a sub-mesoscopic scale. Finally, it should be observed that complementarity is clearly manifest, in a natural manner, in the domain-integrated field equations method, by accounting for the intrinsic complementarity of the physical field quantities pertaining to the static magnetic field when thought of as a pair [6, p. 164]. On the other hand, mixed formulations require a choice for computational complementary quantities to which little (if any) physical meaning can be attributed to. A mutual comparison as to the performance of the different methods is beyond the scope of the present paper.

\section{The Static Magnetic Field Domain-Integrated Field Equations}

The equations to be solved in the static magnetic field domain-integrated field equations method are [1]: the domain-integrated field equations

$$
\int_{\partial \mathcal{D}} \boldsymbol{n} \times \boldsymbol{H} d A=\int_{\mathcal{D}} \boldsymbol{J} d V
$$




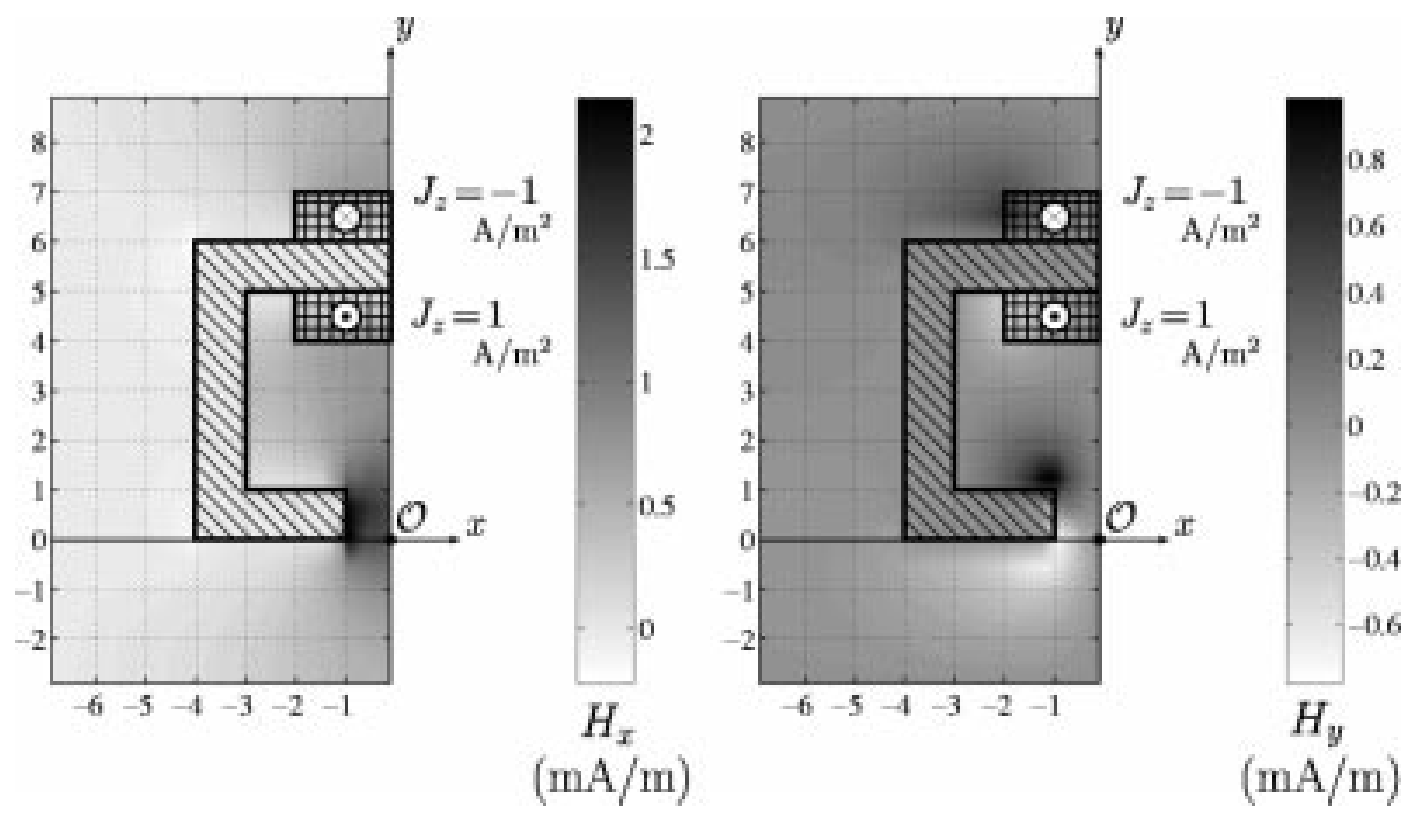

Fig. 5. The plot of the field distribution in the model magnetic head configuration (computational mesh density $14 \times 24$ ).

$$
\int_{\partial \mathcal{D}} \boldsymbol{n} \cdot \boldsymbol{B} d A=0
$$

subject to the compatibility relation

$$
\int_{\mathcal{S}} \boldsymbol{n} \cdot \boldsymbol{J} d A=0
$$

the interface boundary conditions

$$
\begin{gathered}
\boldsymbol{n} \cdot \boldsymbol{J}=0 \text { on boundaries of coils, } \\
\boldsymbol{n} \times \boldsymbol{H}=\text { continuous across interfaces, } \\
\boldsymbol{n} \cdot \boldsymbol{B}=\text { continuous across interfaces, }
\end{gathered}
$$

and the relation

$$
\int_{\mathcal{D}}\left|\boldsymbol{B}-\mu \cdot \boldsymbol{H}-\mu_{0} \boldsymbol{M}\right|^{2} d V=\text { minimum }
$$

In these relations, $J$ is the volume density of impressed electric current, $\boldsymbol{M}$ is the impressed magnetization and $\boldsymbol{\mu}$ is the tensorial permeability. In (1) and (2), $\mathcal{D}$ is any bounded subdomain of $R^{3}, \partial \mathcal{D}$ is its closed boundary and $n$ is the unit vector along the outward normal to $\partial \mathcal{D}$. In $(3), \mathcal{S}$ is any closed surface and $\boldsymbol{n}$ is the unit vector along the outward normal to $\mathcal{S}$. In (4), (5), (6), $\boldsymbol{n}$ denotes the unit vector along the normal to the boundary of coils and to the interfaces, respectively. In the minimization procedure associated with (7), $M$ and $B$ are held fixed and (the expansion of) $\boldsymbol{H}$ is subject to variation.

For the test configurations to be considered, we take $\boldsymbol{J}$ such that (3) and (4) are satisfied, while $\boldsymbol{M}=\mathbf{0}$. Consistently linear edge and face expansion functions are substituted for $\boldsymbol{H}$ and $B$, respectively. Equations (1) and (2) and the minimization in (7) are applied to each triangle of the mesh. Explicit boundary values on the boundary of the domain of computation, either based on the geometrical symmetry or ones that account for almost vanishing values of the tangential part of the magnetic field strength or the normal part of the magnetic flux density, as appropriate, are employed. In this manner, an overdetermined system of linear, algebraic equations in the expansion coefficients of $\boldsymbol{H}$ and $\boldsymbol{B}$ results. This system is subsequently solved by minimizing the $\boldsymbol{L}^{2}$-norm of the residual.

\section{The MOdel Rotor/Stator Slot CONFIGURATION}

The model asynchronous, electrical, rotary machine rotor/stator slot configuration consists of a slot in a highly permeable stator $\left(\mu_{r}=1000\right)$ opposite to an infinitely permeable rotor (see Fig. 1). In the stator slot, an electric current carrying winding is present. The two-component magnetic field strength and magnetic flux density are computed. The configuration is discretized by a uniform mesh consisting of $N \times 2 N,(N=2,4,8,16)$ squares, each of which is divided into four triangles. The residual error in the system of algebraic equations and the number of iterations in the incomplete Cholesky-Conjugate Gradient (IC-CG) process are kept trace of (see Table II), on the basis of which it can be concluded that the numerical problem, as formulated, is well posed. The relatively long computation times are due to the code being implemented entirely in Matlab. Most of the computation time is spent on building the system of equations, a process that involves a large number of loops (structures that are known to be particularly time consuming when implemented in Matlab). Therefore, efficiency can be expected to be subject to considerable improvement. Additionally, the satisfaction of (1) and (2) is tested for the case when $N=8$. The error in the satisfaction of (1) on the integration paths shown in Fig. 3(b) and the normalized fluxes per unit length on the integration paths shown in Fig. 3(c) are shown in Table I. The values of the computed magnetic field strength are shown in Fig. 4. 


\section{The Model Magentic Head Configuration}

The model magnetic recording head configuration consists of a high permeability $\left(\mu_{r}=1000\right)$ cylindrical ring-type head with a gap (see Fig. 2). The head is excited by a single winding electric current carrying coil that is uniform in the direction of cylindricity. The two-component magnetic field strength and magnetic flux density are computed. The configuration is discretized by a uniform mesh consisting of $7 \times 12$ and $14 \times 24$ squares, each of which is divided into four triangles. The residual error in the system of algebraic equations and the number of iterations in the incomplete Cholesky-Conjugate Gradient (IC-CG) process are kept trace of (see Table III). The values of the computed magnetic field strength are shown in Fig. 5.

\section{CONCLUSIONS}

The principles of the domain-integrated field equations approach to static magnetic field computation were discussed, with an emphasis on the natural manner in which they stem from the underlying physics in strongly heterogeneous (and anisotropic) media. The method was applied to some two-di- mensional configurations, with large contrasts in material properties and sharp edges and/or corners. It was shown that all physical laws were satisfied to a uniform degree of (excellent) accuracy, even for coarse meshes.

\section{REFERENCES}

[1] A. T. de Hoop and I. E. Lager, "Static magnetic field computation-an approach based on the domain-integrated field equations," IEEE Transactions on Magnetics, vol. 34, no. 5, pp. 3355-3358, 1998.

[2] G. L. Naber, Topological Methods in Euclidean Spaces. Cambridge: Cambridge University Press, 1980.

[3] P. Alotto, F. Delfino, P. Molfino, M. Nervi, and I. Perugia, "A mixed face-edge finite element formulation for 3D magnetostatic problems," IEEE Transactions on Magnetics, vol. 34, no. 5, pp. 2445-2448, 1998.

[4] P. Dular, J.-F. Remacle, F. Henrotte, A. Genon, and W. Legros, "Magnetostatic and magnetodynamic mixed formulations compared with conventional formulations," IEEE Transactions on Magnetics, vol. 33, no. 2, pp. 1302-1305, 1997.

[5] A. Koski, T. Tarhasaari, and L. Kettunen, "Complementary hybrid formulations for $2 \mathrm{~d}$ magnetostatics," IEEE Transactions on Magnetics, vol. 34, no. 5, pp. 2457-2460, 1998.

[6] A. Bossavit, Computational Electromagnetism. Variational formulations, Complementarity, Edge Elements. San Diego: Academic Press, 1998. 